\title{
KEY KINEMATIC COMPONENTS FOR OPTIMAL BASKETBALL FREE THROW SHOOTING PERFORMAMCE
}

\author{
Dimitrije Cabarkapa $a^{A, B, C, D, E}$ \\ Jayhawk Athletic Performance Laboratory - Wu Tsai Human Performance Alliance, University of Kansas, Lawrence, KS, USA \\ ORCID: 0000-0001-9912-3251 | e-mail: dcabarkapa@ku.edu
}

\author{
Andrew C. Fry ${ }^{A, B, C, D, E}$ \\ Jayhawk Athletic Performance Laboratory - Wu Tsai Human Performance Alliance, University of Kansas, Lawrence, KS, USA \\ ORCID: 0000-0001-8171-7684
}

Kevin M. Carlson ${ }^{A, B}$

College of Arts and Sciences, Des Moines, IA, USA

ORCID: 0000-0003-4154-4664

John P. PoggiOA,C

Department of Educational Psychology, University of Kansas, Lawrence, KS, USA ORCID: 0000-0001-9432-4871

\author{
Michael A. Deane $e^{A, D}$ \\ Jayhawk Athletic Performance Laboratory - Wu Tsai Human Performance Alliance, University of Kansas, Lawrence, KS, USA \\ ORCID: 0000-0002-0954-7649

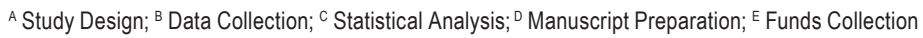

\begin{abstract}
Ahstract The purpose of this study was to determine the difference in five kinematic variables (internal knee and elbow angles, elbow height, forearm angle from vertical, and shoulder flexion angle at ball release) between proficient and non-proficient free throw shooters and which variables had the greatest contributions to a successful free throw shooting outcome. Seventeen male basketball players shoot three sets of 10 free throws with a two-minute break between each set. A three-dimensional motion tracking system composed of 17 sensors sampling at $60 \mathrm{~Hz}$ was used for data collection. Proficient free throw shooters had greater knee and elbow flexion, lower elbow height, and a smaller forearm angle compared to non-proficient shooters. These results explained $89.5 \%$ of the total variance. While maintaining the optimal range of these kinematic variables allows each subject to reach an appropriate level of free throw shooting performance, the key variable capable of distinguishing between made and missed shots within the proficient group of shooters was the forearm angle. Positioning the forearm parallel, or close to parallel, with an imaginary vertical line during the preparatory phase of the shooting motion accounted for $23.9 \%$ of the total variance and was associated with a greater number of made shots.
\end{abstract}

Key WOrlls coaching, sport science, shooting technique, analysis 


\section{Introduction}

Basketball is considered one of the most popular sports worldwide. Fast pace, high scoring, and highly skilled movements significantly contribute to the overall attractiveness of this sport. Since 1891, when James Naismith invented the game of basketball, numerous modifications have been made in order to improve the flow, scoring, and safety of the game while free throw rules have stayed unchanged for almost a century.

Previous research has shown that free throw performance is one of the key factors for determining winning or losing within some of the highest levels of basketball competition (Sampaio, Janeira, 2003). During a regular basketball season, results of four to six games have been solely determined by the in-game players' free throw shooting performance (Hays, Krause, 1987). The team's ability to secure and make more free throw attempts, as well as achieve higher overall shooting percentage, has been correlated with the positive final game outcome (Csatalsay, O'Donoghue, Hughes, Dancs, 2009; Sampaio, Janeira, 2003; Trninic, Dizdar, Luksic, 2002). Additionally, it has been shown that an optimal free throw shooting performance is even more critical close to the end of the game (Sampaio, Janeira, 2003). Currently, based on general coaching norms and previous research findings, it is considered that the average success rate for adult free throw shooting performance is approximately 70\% (Mullineaux, Uhl, 2010; Sampaio, Janeira, 2003; Tran, Silverberg, 2008). Depending on the in-game requirements and the playing style, the percentage of total game points scored from the free throw line can range between 19-25\% (Branch, 2009; Kozar, Vaughn, Whitfield, Lord, Dye, 1994; Sampaio, Janeira, 2003). Hence, considering the impact that successful free throw shooting performance can have on the overall number of scored points and the final game outcome, it is not surprising that basketball coaches dedicate a considerable amount of time to coaching this shooting motion.

Even though the free throw shot has been considered one of the easiest uncontested shooting motions in the game of basketball (Okubo, Hubbard, 2006), many players on various levels of competition struggle with its optimal and consistent execution. A considerable amount of scientific literature has been focused on addressing the optimal free throw shooting trajectory from a theoretical point of view, and its kinematic components that could lead to the successful free throw outcome (Brancazio, 1981; Hamilton, Reinschmidt, 1997; Huston, Grau, 2003; Okubo, Hubbard, 2006; Tan, Miller, 1981; Tran, Silverberg, 2008). Two of the most commonly observed variables necessary for the optimal ball trajectory during a free throw shot include release angle and release speed (Hamilton, Reinschmidt, 1997; Hudson, 1985; Miller, Bartlett, 1996; Tan, Miller, 1981; Tran, Silverberg, 2008). Hamilton and Reinschmidt (1997) found that the optimal release angle and ball speed for a basketball free throw shot should be 60 degrees and $7.3 \mathrm{~m} \cdot \mathrm{s}^{-1}$, respectively. Tan and Miller (1981) obtained similar conclusions regarding the optimal projection velocity magnitudes; however, their theoretical estimations for the angle of basketball projection were lower by approximately nine degrees. Huston and Grau (2003) took a step further with an attempt to theoretically determine the optimal shooting kinematics for various shooting strategies such as free throw, standard shot, and lay-up. The estimated free throw release angle of 51.2 degrees exhibited almost identical agreement with the findings of Tan and Miller (1981), while the minimal release speed was estimated to be $7.14 \mathrm{~m} \cdot \mathrm{s}^{-1}$ (Huston, Grau, 2003). Furthermore, when producing hundreds of thousands of three-dimensional free throw basketball trajectory simulations, Tran and Silverberg (2008) obtained similar findings while adding that if the release angle falls under 52 degrees, the player will be more susceptible to error. Considering that it is almost impossible to produce the identical body motion multiple times in a row, Huston and Grau (2003) estimated the allowable margin of error for each of these variables. They determined that the allowable deviations for the release angle are roughly seven degrees and the ball speed $0.15 \mathrm{~m} \cdot \mathrm{s}^{-1}$ (Huston, Grau, 2003). Thus, failure to achieve these optimal kinematic 
magnitudes and stay within the recommended range could potentially lead to an unsuccessful free throw shooting attempt. While some may assume that these kinematic characteristics are only prominent within high skill basketball players, Hudson's (1985) findings reveal nonexistent differences in both release angle and release speed between low, moderate, and high skill free throw shooters, suggesting that there might be other kinematic contributors affecting the successful execution of the free throw shooting motion.

While the disagreement in release angle and speed between the previously mentioned studies is minimal, the inability to achieve the optimal shooting trajectory may also be attributed to the difference in the estimated average basketball player height incorporated into their theoretical computations. Hamilton and Reinschmidt (1997) indicated that, besides optimal release angle and speed, individual anthropometric characteristics may be another influential factor that influence overall shooting accuracy. For a $6 \mathrm{ft}(182.9 \mathrm{~cm})$ basketball player, the average release height is estimated to be approximately $7 \mathrm{ft}$ (2.134 m) (Huston, Grau, 2003; Tran, Silverberg, 2008). Tran and Silverberg's (2008) findings indicate that greater height of ball release is related to an enhanced basketball shooting performance. Given the equal shooting ability, Brancazio (1981) further supports Tran and Silverberg's findings and denotes that taller players tend to have an advantage in free throw shooting performance due to the enlarged margin of error caused by the higher point of release. It is assumed that these observations may be mainly induced by the lower speed of release and reduced force production requirements (Brancazio, 1981). However, these findings contradict some of the stereotypical beliefs, advocating that taller players tend to be poorer free throw shooters (Robinson, 2016). It is generally assumed that due to a higher release point, a taller player needs to make up for the ball trajectory which requires additional muscular control (Robinson, 2016). While the height contribution to the optimal free throw shooting accuracy is still an underexamined idea, sport scientists need to be conscious of a possibility that designated playing positions more than players' heights are affecting the free throw shooting proficiency. Miller and Bartlett (1996) found more consistent changes in shooting kinematic patterns with adjustments in shooting distances for guards when compared to centers. These findings suggest that shorter players might be more accustomed to shots further away from the basket, such as a free throw, due to their on-court playing requirements (Miller, Bartlett, 1996).

Another factor that has been considered to play an important role for proper free throw shooting mechanics is the backspin. Previous research has found that a free throw shot with more backspin has greater chances to result in a positive outcome (Brancazio, 1981; Hamilton, Reinschmidt, 1997; Okubo and Hubbard, 2006). When a greater margin of error might be created with player's inability to achieve the optimal angle, height or speed at the time point of the ball release, applying an appropriate amount of backspin can serve as a correction factor that may make up for the lack of other kinematic properties (Okubo, Hubbard, 2006). Considering that it is uncommon for an average basketball player to make a series of perfect free throw shots without touching the front or back part of the rim, applying back spin might be a critical component. Based on the laws of physics, when a basketball with an appropriate amount of backspin touches the rim, it will alter its translational and rotational motions (Brancazio, 1981). Due to this effect, the ball can roll into the rim even though the shot trajectory was not ideal (Brancazio, 1981). Several other reports further support the importance of the backspin while suggesting that players should aim closer to the back of the rim as this approach can elicit a higher free throw shooting accuracy (Hamilton, Reinschmidt, 1997; Okubo, Hubbard, 2006; Tran, Silverberg, 2008). Additionally, while backspin is important for proper shooting trajectory, the force exerted on a rapidly spinning cylinder through the air in a direction at an angle to the axis of spin, known as the Magnus effect, could potentially influence the optimal free throw trajectory. Huston and Grau 
(2003) estimated that the Magnus effect with a $7.14 \mathrm{~m} \cdot \mathrm{s}^{-1}$ ball release speed, is capable of exerting force about $4 \%$ of the overall basketball weight. Although, when considering that average time for free throw shot is approximately one second, the effect of air resistance within this small amount of time might not be capable of eliciting significant modifications to the optimal ball trajectory (Brancazio, 1981).

Based on the previously mentioned research findings, we understand that there is a considerable amount of scientific literature addressing some of the major factors necessary for the optimal free throw shooting trajectory. While their validity and importance remain intact and unchallenged, the practical segment of sports science that addresses the influence of some of the highly emphasized coaching cues for kinematic variables that players can instantaneously implement to improve free throw shooting performance is almost non-existent. Hence, the purpose of this study was to investigate the difference in these kinematic variables between proficient and non-proficient free throw shooters, as well as to determine which variables have the greatest contribution to the successful free throw shooting outcome.

\section{Methods}

\section{Subjects}

Seventeen healthy recreationally active male basketball players (height $=182.7 \pm 8.9 \mathrm{~cm}$, weight $=88.9$ $\pm 6.5 \mathrm{~kg}$, age $=29.6 \pm 10.1$ years) volunteered to participate in this research study. Each participant completed the informed consent form prior to any testing procedures. Participants with any musculoskeletal injuries were not permitted to participate in this study. All procedures performed in this study were previously approved by the University's Institutional Review Board.

\section{Procedures}

Upon arrival to the testing facility, participants performed a standardized warm-up procedure consisting of a five-minute treadmill run at a moderate intensity and a set of dynamic exercises involving high knees, buttkicks, lunge-and-twist, lateral slides, high skips, and lateral lunges. Each participant was individually familiarized with a three-dimensional motion tracking system (XSENS MVN Awinda, Enschelde, Netherlands) used for the data collection. The motion tracking system, sampling at $60 \mathrm{~Hz}$, contained 17 wireless sensors secured with a specially designed tight Lycra suit and a set of mounting Velcro straps. Each sensor was composed of an accelerometer, gyroscope, magnetometer, and barometer. All sensors were individually placed based on the manufacturer's instructions on the following body locations: left and right foot (middle of the bridge of foot), left and right shank (anterior surface of the tibia), left and right lateral mid-thigh (superior to the knee), pelvis (posterior sacrum), sternum (middle of the chest), left and right shoulder (mid-scapula), left and right upper arm (lateral side superior to the elbow), left and right posterior forearm (proximal to the wrist), left and right posterior hand, and head (middle of the forehead by frontal eminence). After placement of the sensors, the motion tracking system was calibrated following the detailed instructions provided by the manufacturer. Each participant was provided with a size 7 standardized basketball $(75 \mathrm{~cm})$, while the goal was positioned at a standardized height of 10 feet $(3.05 \mathrm{~m})$. The experimental set-up is shown in Figure 1. Participants shot three sets of 10 free throws with a two-minute break between each set to assure adequate recovery. Throughout all testing procedures, a rebounder was present in order to preserve participant's energy and assure an optimal focus directed towards the free throw shooting motion. A research 
assistant kept track of the overall number of free throw shots that the participant took, as well as which specific shots were missed. To eliminate any possible distractions caused by other participants in the testing facility, each participant individually performed the testing procedures. Recorded performance variables include number of made and missed free throw shots, and the percentage of made free throws. Participants making $\geq 70 \%$ of their free throw shots were categorized as "proficient", and those making < $70 \%$ were categorized as "non-proficient", based on the feedback from our panel of experts.

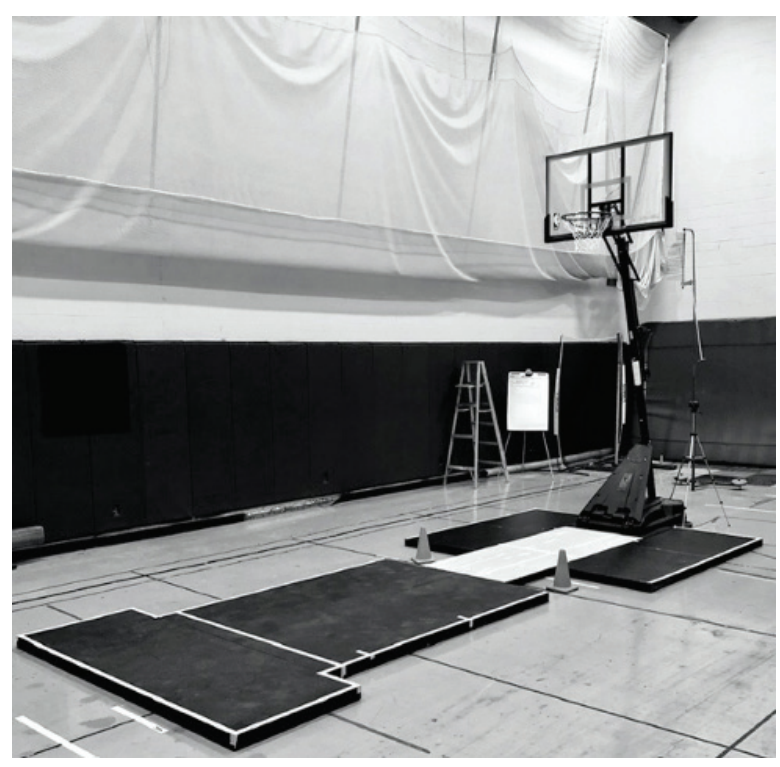

Figure 1. Experimental set-up

\section{Variables}

This study was focused on five free throw kinematic variables, selected after extensive conversations with an expert panel of highly reputable basketball coaches, and obtaining their input regarding key factors considered crucial for the optimal free throw shooting performance. The first dependent variable is the knee angle (Figure 2, angle a) which is defined as the maximum internal angle at the knee joint during the preparatory phase of the free throw shooting motion. The second dependent variable is the elbow height (Figure 2, line b), defined as the perpendicular distance between the olecranon process and the ground immediately prior to the free throw initiation. The third dependent variable is elbow angle (Figure 2, angle c), defined as the internal angle of the elbow at the initial stage of the free throw shooting motion. The fourth dependent variable is the forearm angle (Figure 2, angle d), defined as the angle between the long axis of the forearm and an imaginary vertical line at the initiation of the free throw shooting motion. This variable is used to determine the magnitude of the lateral elbow deviation. If the forearm is parallel with the imaginary vertical line, the forearm angle value will be equal to $0^{\circ}$. The fifth dependent variable is the shoulder angle (Figure 2, angle e), which is the angle between the long axis of the arm segment and 
an imaginary vertical line that passes right through the center of the glenohumeral joint at the time point of the ball release. The value for the shoulder angle is equal to $0^{\circ}$ when an individual is standing in the standard anatomical position with the upper limbs parallel to the torso.

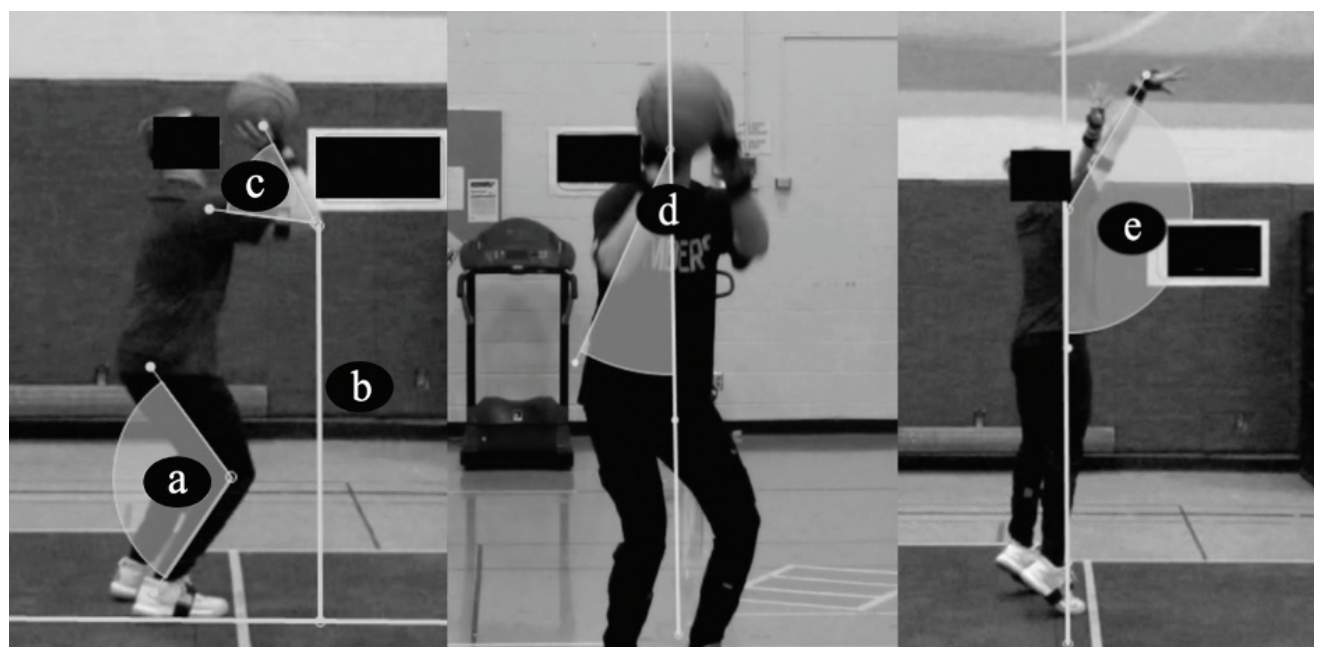

Figure 2. Graphical representation of measured dependent variables: (a) knee angle, (b) elbow height, (c) elbow angle, (d) forearm angle, (e) shoulder angle

\section{Statistical Analysis}

Descriptive statistics and standard deviations $(\bar{x} \pm S D$ ) were calculated for each of the dependent variables. A multivariate Hotelling's T-Squared test was used to detect the difference in the dependent variables between proficient $(\geq 70 \%)$ and non-proficient $(<70 \%)$ free throw shooters. Although the purpose of this study was not to develop a prediction equation and determine the optimal shooting kinematics, a full-model discriminant function analysis was used to quantify the contribution of each of the dependent variables separating proficient from non-proficient free throw shooters. Additionally, a multivariate Hotelling's T-Squared test was used to determine the difference in the dependent variables between made and missed free throw shots within just the group of proficient free throw shooters $(\geq 70 \%)$. Levene's test was used to examine if the data sets met the homogeneity of variance assumption. Statistical significance was set a priori to $p<0.05$. All statistical analyses were completed with SPSS Version 25.0 software statistical package (SPSS Inc. Chicago, IL, USA).

\section{Results}

Mean values and standard deviations ( $\bar{x} \pm S D$ ) for each of the dependent variables for both proficient $(\geq 70 \%)$ and non-proficient $(<70 \%)$ free throw shooters are presented in Table 1 . The average free throw shooting percentage for proficient and non-proficient group of free throw shooters was $82.7 \pm 7.9 \%$ and $52.4 \pm 13.4 \%$, respectively. The total number of free throw shots that subjects performed was 495, from which 295 shots have been attempted 
by proficient and 200 by non-proficient free throw shooters. In order to assure equal sample sizes and avoid violation of homogeneity of variance, 95 samples from the proficient group of shooters have been randomly removed by the SPSS software. Hotelling's T-Squared test indicated a highly significant difference $(p<0.001)$ between all of the dependent variables examined in this study, except for the shoulder angle variable $(p>0.05)$.

Tahle 1. Mean and standard deviations $(\bar{x} \pm$ SD) for all dependent variables between proficient $(\geq 70 \%)$ and non-proficient $(<70 \%)$ free throw shooters

\begin{tabular}{lcc}
\hline \multicolumn{1}{c}{ Dependent variables } & Proficient shooters & Non-proficient shooters \\
\hline Knee flexion $(\mathrm{deg})$ & $108.5 \pm 9.8$ & $117.9 \pm 16.3^{*}$ \\
Elbow height $(\mathrm{cm})$ & $147.6 \pm 9.3$ & $153.4 \pm 16.5^{*}$ \\
Elbow angle $(\mathrm{deg})$ & $71.9 \pm 5.6$ & $80.5 \pm 6.3^{*}$ \\
Forearm angle $(\mathrm{deg})$ & $7.9 \pm 7.2$ & $19.8 \pm 17.6^{*}$ \\
Shoulder angle $(\mathrm{deg})$ & $123.4 \pm 15.3$ & $124.4 \pm 14.2$ \\
\hline
\end{tabular}

Significant difference $(p<0.05)$.

Based on the standardized discriminant function analysis weights, the highest prominence in predicting proficient vs. non-proficient free throw shooters was attributed to the elbow angle and forearm angle variables. The magnitudes of standardized discriminant function coefficients (beta weights), and the percentage of total and explained variance for each of the dependent variables examined in this study are presented in Table 2. The computed discriminant function analysis was able to correctly classify free throw shooters in the proficient or non-proficient category based on the observed dependent variables in $89.5 \%$ cases. The detailed classification results for predicted group membership are presented in Table 3.

Tahle 2. Standardized discriminant function coefficients, and the percentage of explained and total variance for each of the dependent variables examined in this study

\begin{tabular}{lccc}
\hline \multicolumn{1}{c}{ Dependent variables } & Standardized coefficients & Percentage of explained variance & Percentage of total variance \\
\hline Knee flexion $(\mathrm{deg})$ & -0.091 & 3.2 & 2.9 \\
Elbow height $(\mathrm{cm})$ & -0.604 & 21.5 & 19.2 \\
Elbow angle $(\mathrm{deg})$ & 1.151 & 41.0 & 36.7 \\
Forearm angle $(\mathrm{deg})$ & 0.750 & 26.7 & 23.9 \\
Shoulder angle $(\mathrm{deg})$ & -0.213 & 7.6 & 6.8 \\
\hline Total & & 100.0 & 89.5 \\
\hline
\end{tabular}

Canonical correlation $=0.723($ effect size $=0.523)$.

Table 3. Classification results for predicted group membership

\begin{tabular}{|c|c|c|c|}
\hline \multirow{2}{*}{ Actual Group } & \multicolumn{2}{|c|}{ Predicted group membership } & \multirow{2}{*}{ Number of cases } \\
\hline & non-proficient & proficient & \\
\hline Non-proficient & $176(88 \%)$ & $24(12 \%)$ & 200 \\
\hline Proficient & $18(9 \%)$ & $182(91 \%)$ & 200 \\
\hline
\end{tabular}

$89.5 \%$ of subjects correctly classified $(p<0.05)$. 
Mean values and standard deviations $(\bar{x} \pm S D)$ for each of the dependent variables between made and missed free throw shots within the proficient $(\geq 70 \%)$ group of shooters are presented in Table 4 . The total number of made and missed free throw shots was 157 and 43, respectively. Levene's test did not reach the level of statistical significance $(p>0.05)$ for all of the variables examined in this study. The only statistically significant difference in the dependent variables between made and missed free throw shots within a group of proficient free throw shooters was present for the forearm angle variable $(p=0.004)$.

Table 4. Mean and standard deviations $(\bar{x} \pm S D)$ for all dependent variables between made and missed free throw shots within proficient $(\geq 70 \%)$ free throw shooters

\begin{tabular}{lcc}
\hline \multicolumn{1}{c}{ Dependent variables } & Made shots & Missed shots \\
\hline Knee flexion (deg) & $108.1 \pm 10.1$ & $110.0 \pm 8.3$ \\
Elbow height (cm) & $147.7 \pm 9.2$ & $147.2 \pm 9.9$ \\
Elbow angle (deg) & $72.3 \pm 5.3$ & $70.5 \pm 6.5$ \\
Forearm angle (deg) & $7.2 \pm 5.6$ & $10.7 \pm 10.1^{\circ}$ \\
Shoulder angle (deg) & $123.5 \pm 15.8$ & $122.7 \pm 13.7$ \\
\hline
\end{tabular}

"Significant difference $(p<0.05)$.

\section{Discussion}

Based on the findings of this study, we identified some distinguishable kinematic differences between proficient and non-proficient free throw shooters. Proficient free throw shooters implemented some of the highly emphasized coaching cues during the preparatory phase of the shooting motion, which ultimately lead to increased shooting accuracy. Considering that knee flexion allows for the optimal lower body leverage and power contribution, the proficient group of shooters had significantly lower knee angles values compared to the non-proficient group. Despite the minimal or almost non-existent impact on the overall shooting ability prediction model, greater knee flexion during the preparatory phase of the free throw shooting motion may play an important role in achieving the desired level of free throw shooting accuracy. Our observations are in agreement with the findings of Cabarkapa, Fry, Poggio, Deane (2021) that used video analysis to examine kinematic differences between proficient and nonproficient free throw shooters. In another investigation, the researchers suggested that greater total movement in the knee joint was one of the critical kinematic components for successful free throw shot (Ammar, Chtourou, Abdelkarim, Parish, Hoekelmann, 2016). Unlike observed in the present study, lower flexion in the preparatory phase and greater extension at the release phase of the shooting motion were related to improvements in player's shooting technique and ultimately lead to a greater number of made free throws (Ammar et al., 2016). However, when the difference in the knee angle between made and missed free throw shots was examined within a group of proficient shooters, the knee angle did not demonstrate statistical significance. Similar findings were reported by Uygur, Goktepe, Ak, Karabork, Korkusuz (2010) when they examined the effect of fatigue on kinematics of basketball free throw shooting technique. They found that the knee angle before, after, and at the ball release was not significantly different between made and missed free throw shots (Uygur, Goktepe, Ak, Karabork, Korkusuz, 2010). The inability to observe a difference in knee flexion may be mainly attributed to the cohort of subjects that volunteered to participate in the study. Uygur at al.'s (2010) observations were based on a group of high-level collegiate athletes 
that are assumed to have a respectable average free throw percentage, which based on their free throw shooting skill ability, might be comparable to the proficient group of shooters tested in the present study.

Two dependent variables that demonstrated the greatest impact in predicting between proficient and nonproficient free throw shooters were elbow flexion and forearm angle. Proficient shooters had greater elbow flexion and less lateral elbow deviation from the imaginary vertical axis when compared to the non-proficient group of free throw shooters. While to date, no research has specifically focused on the importance of these variables for proper shooting mechanics and their influence on the optimal shooting trajectory, Mullineaux and Uhl (2010) examined the coordination-variability and kinematic differences between made and missed free throw shots within a cohort of elite collegiate basketball players. They found that the elbow kinematics influenced the wrist mechanics though velocity-dependent-torques (Mullineaux, Uhl, 2010). Although Miller and Bartlett (1996) focused on examining the kinematic differences between short, mid-range, and long-range basketball shots through high-speed videography, their findings further support the importance of the elbow-wrist alignment and indicate that elbow extension for both guards and forwards was related to the increased release speed of the ball. Hence, considering the importance of proper backspin during the release phase of the free throw shot (Brancazio, 1981; Hamilton, Reinschmidt, 1997; Okubo, Hubbard, 2006), we can assume that our data builds upon the previously mentioned literature. Proper elbow positioning may allow for the optimal amount of backspin applied on the ball through proper kinematic chaining at the time point of ball release. Greater elbow flexion can potentially increase the force production necessary to push the ball away from the body while minimal to no forearm deviation would assure that the applied force is completely transmitted through the imaginary vertical axis. Ultimately, failure to achieve the previously mentioned kinematic parameters may impair achieving optimal ball release velocity and height, which has been previously determined as the vital components for the successful outcome of the free throw shot (Hamilton, Reinschmidt, 1997; Hudson, 1985; Miller, Bartlett, 1996; Tan, Miller, 1981; Tran, Silverberg, 2008).

Considering that it is necessary for proficient free throw shooters to maintain all of the other kinematic variables examined in this study within the optimal range, an inability to position the forearm parallel, or as close to parallel, with an imaginary vertical line was as a key factor contributing to an unsuccessful shooting outcome. The difference in the forearm angle between made and missed shooting attempts observed within a proficient group of free throw shooters was minimal; approximately three degrees. While successful execution of the free throw shooting motion requires precise movements influenced by endless number of body kinematic combinations (Hudson, 1985), previously conducted research indicated that margin of error that can distinguish between made and missed free throw shots is very small (Mullineaux, Uhl, 2010). In order to assure that these conditions have been met, we may be able to focus on another variable observed in this study, the elbow height. It is logical that the player's ability to achieve greater elbow flexion and decrease the amount of forearm medial or lateral deviation will lead to lower elbow height. Our findings entirely support these assumptions, indicating that the subjects in the proficient group of shooters had significantly lower elbow heights during the preparatory phase of the free throw shooting motion when compared to the non-proficient group of shooters. While the magnitude of difference was evident, the elbow height demonstrated negative moderate to strong contribution to predicting the player's free throw shooting proficiency. This signifies that higher elbow positioning might be related to increased chances of negative free throw shooting outcome. Thus, based on these findings we may assume that the elbow height might 
serve as a rapid estimate for the proper elbow positioning during the preparatory phase of the free throw shooting motion that can be quickly assessed by basketball coaching personnel in a practical setting.

While all of the previously mentioned kinematic variables were significantly different between the proficient and non-proficient group of free throw shooters, the only variable with almost nonexistent difference was the shoulder angle. Our results revealed similar values when compared to the findings of Miller and Bartlett (1996), even though they reported shoulder angle values at the time-point of the ball release for mid-range shots within the free throw shooting line distance. Interestingly, while Goosey-Tolfrey, Butterworth and Morriss (2002) analyzed upper-body kinematics of the free throw shot within a cohort of wheelchair basketball players, the shoulder angle at the ball release detected was almost identical to the values observed in this study. It was suggested that less shoulder flexion leads to greater wrist velocity compensation (Goosey-Tolfrey, Butterworth, Morriss, 2002). Based on these findings we can be assume that despite the inability to observe the difference in the shoulder angle variable between the proficient and non-proficient groups of free throw shooters, both groups were able to attain the optimal shoulder angle magnitudes. Similar findings from Ammar et al. (2016) study focused on examining the difference in shoulder angle at the time-point of ball release between made and missed free throw shots. While the shoulder angle was not significantly different in the present and in the previously mentioned studies, we should not assume that this variable is unnecessary for the successful shooting outcome, especially when considering the slight negative influence of this variable on the free throw outcome prediction model. Thus, we may want to treat this variable as one of the first and most commonly adjusted factors needed to achieve the optimal release angle and velocity.

While all of the previously mentioned variables exhibited significant and a considerable contribution to overall free throw shooting skill prediction, we need to be cautious when interpreting these results. The findings of this study need to be interpreted more as a set of key interdependent kinematic variables, than solely focus on one specific variable. Even though players may be capable of achieving an optimal forearm angle, an inability to control for the optimal magnitudes of the other key kinematic components during the preparatory and completion phase of the shooting motion can result in unsuccessful shooting performance. Considering that this was the first study focused on investigating the influence of some of the highly emphasized free throw coaching cues on the success of the free throw shooting performance, further research is needed to examine the relationship between the variables examined in this study and their effect on the optimal shooting trajectory. Moreover, while our observations were solely based on a cohort of recreationally active basketball players, further research should focus on examining the difference in the same kinematic variables within diverse levels of basketball playing competitions such as high school, collegiate, and professional.

\section{Conclusion}

Based on the findings of this study, we can conclude that common coaching cues addressing the proper preparatory and completion phases of the free throw shooting motion can significantly influence the positive outcome of the shot and successfully distinguish between proficient and non-proficient free throw shooters. Proficient free throw shooters exhibited greater knee and elbow flexion, lower relative elbow height, and smaller forearm angle values relative to vertical. While maintaining the optimal range of these kinematic variables can allow a player to reach an acceptable level of free throw shooting performance, the key variable capable of distinguishing between made and missed shots within the proficient group of shooters was forearm angle. The ability to position the forearm 
parallel, or close to parallel with the imaginary vertical line during the preparatory phase of the shooting motion may result in a greater number of made free throw shots.

\section{References}

Ammar, A., Chtourou, H., Abdelkarim, O., Parish, A., Hoekelmann, A. (2016). Free throw shot in basketball: kinematic analysis of scored and missed shots during the learning process. Sport Sciences for Health, 12 (1), $27-33$.

Branch, J. (2009, March 04). For free throws, 50 years of practice is no help. Retrieved from: https://www.nytimes.com/2009/03/04/ sports/basketball/04freethrow.html (2.07.2020).

Brancazio, P.J. (1981). Physics of basketball. American Journal of Physics, 49 (4), 356-365.

Cabarkapa, D., Fry, A.C., Poggio, J.P, Deane, M.A. (in press). Kinematic differences between proficient and non-proficient free throw shooters - a video analysis. Journal of Applied Sport Sciences.

Csataljay, G., O'Donoghue, P., Hughes, M., Dancs, H. (2009). Performance indicators that distinguish winning and losing teams in basketball. International Journal of Performance Analysis in Sport, 9 (1), 60-66.

Goosey-Tolfrey, V., Butterworth, D., Morriss, C. (2002). Free throw shooting technique of male wheelchair basketball players. Adapted Physical Activity Quarterly, 19 (2), 238-250.

Hays, D., Krause, J. V. (1987). Score on the Throw. The Basketball Bulletin, Winter, 4-9.

Huston, R.L., Grau, C.A. (2003). Basketball shooting strategies - the free throw, direct shot and layup. Sports Engineering, 6(1), $49-64$. Hamilton, G.R., Reinschmidt, C. (1997). Optimal trajectory for the basketball free throw. Journal of Sports Sciences, 15 (5), $491-504$.

Hudson, J.L. (1985). Prediction of basketball skill using biomechanical variables. Research Quarterly for Exercise and Sport, 56 (2), 115-121.

Kozar, B., Vaughn, R.E., Whitfield, K.E., Lord, R.H., Dye, B. (1994). Importance of free-throws at various stages of basketball games. Perceptual and Motor Skills, 78 (1), 243-248.

Mullineaux, D.R., Uhl, T.L. (2010). Coordination-variability and kinematics of misses versus swishes of basketball free throws. Journal of Sports Sciences, 28 (9), 1017-1024.

Miller, S., Bartlett, R. (1996). The relationship between basketball shooting kinematics, distance and playing position. Journal of Sports Sciences, 14 (3), 243-253.

Okubo, H., Hubbard, M. (2006). Dynamics of the basketball shot with application to the free throw. Journal of Sports Sciences, 24 (12), 1303-1314.

Robinson, N. (2016, July 25). Why are NBA big men so useless at free throws? Retrieved from: http://theniche-cache.com/americansports/why-are-nba-big-men-so-useless-at-free-throws (2.07.2020).

Sampaio, J., Janeira, M. (2003). Importance of free-throw performance in game outcome during the final series of basketball playoffs. International Journal of Applied Sports Sciences, 15 (2), 9-16.

Trninic, S., Dizdar, D., Luksic, E. (2002). Differences between winning and defeated top quality basketball teams in final tournaments of European club championship. Collegium Antropologicum, 26 (2), 521-531.

Tran, C.M., Silverberg, L.M. (2008). Optimal release conditions for the free throw in men's basketball. Journal of Sports Sciences, 26 (11), 1147-1155.

Tan, A., Miller, G. (1981). Kinematics of the free throw in basketball. American Journal of Physics, 49 (6), 542-544.

Uygur, M., Goktepe, A., Ak, E., Karabork, H., Korkusuz, F. (2010). The effect of fatigue on the kinematics of free throw shooting in basketball. Journal of Human Kinetics, 24 (2010), 51-56.

Cite this anticle as: Cabarkapa, D., Fry, A.C., Carlson, K.M., Poggio, J.P., Deane, M.A. (2021). Key Kinematic Components for Optimal Basketball Free Throw Shooting Performance. Central European Journal of Sport Sciences and Medicine, 4 (36), 5-15. DOI: 10.18276/cej.2021.4-01. 